\title{
Adsorption of Charged Protein Residues on an Inorganic Nanosheet: Computer Simulation of LDH Interaction with Ion Channel
}

\author{
Alexey A. Tsukanov ${ }^{1, a)}$ and Sergey G. Psakhie ${ }^{2,3, b)}$ \\ ${ }^{1}$ Skolkovo Institute of Science and Technologies, Moscow, 143025 Russia \\ ${ }^{2}$ National Research Tomsk Polytechnic University, Tomsk, 634050 Russia \\ ${ }^{3}$ Institute of Strength Physics and Materials Science SB RAS, Tomsk, 634055 Russia

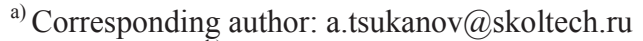 \\ ${ }^{b)} \mathrm{sp} @$ ispms.tsc.ru
}

\begin{abstract}
Quasi-two-dimensional and hybrid nanomaterials based on layered double hydroxides (LDH), cationic clays, layered oxyhydroxides and hydroxides of metals possess large specific surface area and strong electrostatic properties with permanent or $\mathrm{pH}$-dependent electric charge. Such nanomaterials may impact cellular electrostatics, changing the ion balance, $\mathrm{pH}$ and membrane potential. Selective ion adsorption/exchange may alter the transmembrane electrochemical gradient, disrupting potential-dependent cellular processes. Cellular proteins as a rule have charged residues which can be effectively adsorbed on the surface of layered hydroxide based nanomaterials. The aim of this study is to attempt to shed some light on the possibility and mechanisms of protein "adhesion" an LDH nanosheet and to propose a new direction in anticancer medicine, based on physical impact and strong electrostatics. An unbiased molecular dynamics simulation was performed and the combined process free energy estimation (COPFEE) approach was used.
\end{abstract}

\section{INTRODUCTION}

The attention to clay minerals in medical applications dates back very far [1-3]. In recent decades inorganic twodimensional nanomaterials and bio-nanohybrids based on clay minerals have been employed for targeted drug and gene delivery with controlled release $[4,5]$, medical imaging and protein separation [6], which is an intensively developing field in nanomedicine, diagnostics and pharmaceutics [7, 8]. Hierarchical nanoparticles with an external shell made of layered hydroxides and oxyhydroxides of metals are used as multifunctional bioactive agents for biomedical purposes $[9,10]$ including anti-cancer therapy $[11,12]$.

Clay minerals have layered structure and can be formally divided into three groups depending on the sign of the charge of the pristine nanosheet: neutral - nonionic clays (nanolayers may be glued together by hydrogen bonds), negative - cationic clays (negatively charged layers glued by intercalated cationic species), positive - anionic clays (layered double hydroxide (LDH) subfamily). Hosted hydroxide nanosheets of anionic and cationic clays can strongly adsorb anions and cations, respectively, due to their electric charge. Thus, they can be considered as an effective adsorbent and exchanger of organic anions and ions of biological environments, including charged and polar fragments of proteins, e.g. anionic aspartic and glutamic amino acids or cationic arginine, lysine and protonated histidine.

The molecular dynamics (MD) study presented here is an attempt to understand possible molecular mechanisms of the interaction of the LDH nanosheet with membrane proteins due to competitive adsorption of charged amino acid residues in the presence of extracellular anions. If the adsorption of polar and charged fragments of proteins is sufficiently strong, this may disrupt nutrition and signaling processes of a cell, so the strong surface charge and anion exchange capacity of LDH may become a new tool for cancer medicine, where selective targeting can be provided by using hierarchical ferromagnetic-LDH nanohybrids etc.

Physics of Cancer: Interdisciplinary Problems and Clinical Applications (PC'16)

AIP Conf. Proc. 1760, 020066-1-020066-6; doi: 10.1063/1.4960285

Published by AIP Publishing. 978-0-7354-1418-1/\$30.00 


\section{RESULTS AND DISCUSSION \\ Combined Process Free Energy Estimation}

First we present the relatively fast computational technique we used to estimate the free energy of adsorption, which is based on the potential of mean force (PMF) analysis [13].

Consider an NPT thermodynamic system, which is usually used for molecular dynamics simulations. The change $\Delta G_{\text {sys }}$ of the free energy of the system is less than or equal to work done by some external force acting to on system [14]

$$
\Delta G_{\text {sys }} \leq A_{\text {ext }} .
$$

In case of an irreversible process, entropy generation $\delta S_{\text {gen }}>0$ occurs. The inequality (1) can be rewritten in equation form as

$$
\Delta G_{\text {sys }}+T \delta S_{\text {gen }}=A_{\text {ext }} .
$$

To estimate the free energy of adsorption of some molecule from the bulk water solution onto the sorbent surface, let us consider the following combination of two processes:

1. Forward process - forced adsorption, in which an external force is performing the work for a molecule translocation from the bulk water solution to the surface of the sorbent

$$
\Delta G_{\mathrm{ads}}+T \delta S_{\mathrm{gen}}^{\text {forward }}=A_{\mathrm{ext}}^{\text {forward }} .
$$

2. Reverse process - forced desorption. An external force acts to remove the adsorbed molecule from the sorbent surface into the solvent ( $T$ is constant and equal to $T$ of the forward process)

$$
-\Delta G_{\mathrm{ads}}+T \delta S_{\mathrm{gen}}^{\text {reverse }}=A_{\mathrm{ext}}^{\text {reverse }}
$$

Combining both equations (3) and (4), the following relation can be obtained for an adsorption-desorption pseudo-cycle:

$$
\Delta G_{\mathrm{ads}}=\frac{A_{\mathrm{ext}}^{\text {forward }}-A_{\mathrm{ext}}^{\text {reverse }}}{2}-\frac{T}{2}\left(\delta S_{\mathrm{gen}}^{\text {forward }}-S_{\mathrm{gen}}^{\text {reverse }}\right)
$$

Let us further assume that both the forward and reverse processes are constant velocity pulling procedures (i.e. cv-SMD [13]) with similar and sufficiently small velocities, so that the entropy generation of both stages is approximately equal

$$
\delta S_{\text {gen }}^{\text {forward }} \cong S_{\text {gen }}^{\text {reverse }} .
$$

In that case the second term in (5) falls away and the following approximation results

$$
\Delta G_{\mathrm{ads}} \cong \frac{A_{\mathrm{ext}}^{\text {forward }}-A_{\mathrm{ext}}^{\text {reverse }}}{2} .
$$

In case of ordinary adsorption, when the adsorbed state is energetically favorable and corresponds to a local free energy minimum, the next three inequalities are satisfied (Fig. 1)

$$
\begin{gathered}
A_{\text {ext }}^{\text {forward }}<0, \\
A_{\text {ext }}^{\text {reverse }}>0, \\
\left|A_{\text {ext }}^{\text {forward }}\right| \leq\left|A_{\text {ext }}^{\text {reverse }}\right| .
\end{gathered}
$$

Taking this into account, the relation (7) can be rewritten as

$$
\Delta G_{\mathrm{ads}}^{*}=\frac{-\left|A_{\mathrm{ext}}^{\text {forward }}\right|-\left|A_{\mathrm{ext}}^{\text {reverse }}\right|}{2}=-\frac{1}{2}\left(\left|A_{\mathrm{ext}}^{\text {forward }}\right|+\left|A_{\mathrm{ext}}^{\text {reverse }}\right|\right) .
$$

If forward and reverse pulling processes are reversible then the entropy generation is zero $\delta S_{\text {gen }}=0$ and absolute values of the work performed by the external force are equal $\left|A_{\text {ext }}^{\text {forward }}\right|=\left|A_{\text {ext }}^{\text {reverse }}\right|=|\Delta G|$. The irreversible part may be roughly evaluated as the half-width of the "corridor" between the forward and reverse PMF estimations. 


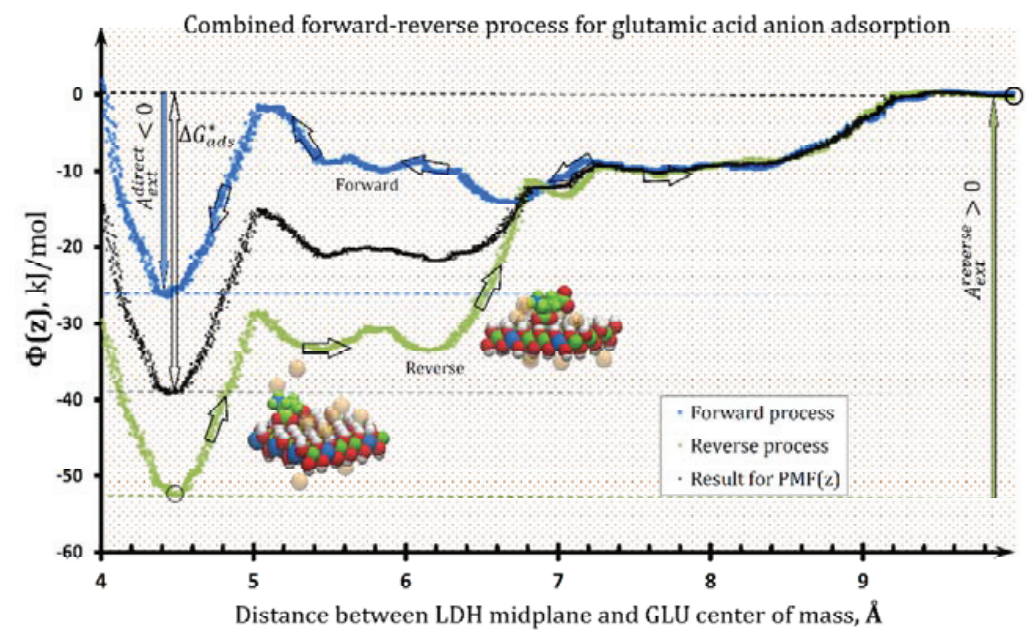

FIGURE 1. PMF analysis for the combined forward-reverse process of glutamic amino acid anion adsorption on the $\mathrm{Mg} / \mathrm{Al}-\mathrm{LDH}$ surface. Blue curve is the PMF profile evaluated for the forward process of forced adsorption, green curve is the PMF profile for the reverse process of forced desorption. The result of the COPFEE procedure is represented by the black curve. The inserts show the typical GLU anion conformations during adsorption/desorption (green van der Waals spheres with red oxygen atoms). Semi-transparent yellow spheres represent chloride ions.

Water is not shown

\section{Competitive/Selective Adsorption of Organic Anions on LDH in the Presence of $\mathrm{Cl}^{-}$ions}

In the framework of this study the glutamic amino acid residue (GLU) was chosen for the investigation of adsorption, since GLU is an anionic compound and is one of the twenty building blocks of the proteins. A positively charged $\mathrm{Mg} / \mathrm{Al}-\mathrm{LDH}$ nanosheet was chosen as a representative of anionic clay minerals, since it has a well-known crystal structure [15]. Chloride ions are the most prevalent anions of the extracellular medium, and tend to be concentrated near the positively charged nanosheet and to screen thereby screen its surface charge.

Conducting a cv-SMD $(v=0.2 \AA / \mathrm{ns})$ simulation for the combined process of GLU adsorption/desorption on $\mathrm{Mg} / \mathrm{Al}-\mathrm{LDH}$ in the presence of chloride ions it was found that the external work in the forward process is $A_{\mathrm{ext}}^{\text {forward }}=-27 \mathrm{~kJ} / \mathrm{mol}$, and in the reverse process $A_{\mathrm{ext}}^{\text {reverse }}=53 \mathrm{~kJ} / \mathrm{mol}$. Employing the relation (11) the following estimation for the free energy of the (competitive) adsorption is obtained: $\Delta G_{\mathrm{ads}}^{*}=-40 \mathrm{~kJ} / \mathrm{mol}$.

The result obtained with this COPFEE procedure is in sufficiently good agreement with the result of SMD simulation from Ref. [16] obtained via multiple PMF evaluation, $\Delta G_{\text {ads }}^{\mathrm{comp}} \approx \Delta G_{\text {ads }}^{\mathrm{GLU}}-\Delta G_{\mathrm{ads}}^{\mathrm{Cl}}=(-53.3 \pm 10.9)-(-8.7$ $\pm 1.8)=-44.6 \pm 12.7 \mathrm{~kJ} / \mathrm{mol}$. Thus, the proposed COPFEE procedure may give an acceptable estimation of the free energy adsorption for small molecules.

The obtained value $\Delta G_{\text {ads }}^{*}=-40 \mathrm{~kJ} / \mathrm{mol}$ for the free energy of competitive adsorption of GLU by Mg/Al-LDH in the presence of $\mathrm{Cl}^{-}$indicates that GLU residues will bind with the LDH surface exchanging $\mathrm{Cl}^{-}$anions. Glutamic amino acid is a building block for many polypeptides and proteins, which is why it can be supposed that protein segments containing this residue may be adsorbed by $\mathrm{Mg} / \mathrm{Al}-\mathrm{LDH}$ nanosheets as well. This effect of LDH may disturb the functioning of proteins and coupled cellular processes, and especially membrane proteins acting as ion channels.

\section{Layered Double Hydroxide Nanosheet Interaction with Ion Channel}

A short molecular dynamics study of $\sim 0.1 \mu$ s was performed to investigate the interaction of an extracellular portion of an ion channel with a fragment of an $\mathrm{Mg} / \mathrm{Al}$ layered double hydroxide nanosheet. A bacterial voltage gated sodium channel $\mathrm{Na}_{\mathrm{V}} \mathrm{Ab}$ embedded into a POPC lipid bilayer was chosen for the model, since their all-atom structure is well-studied $[17,18]$. The presence of the hosted POPC bilayer provides a suitable environment for the membrane protein. 


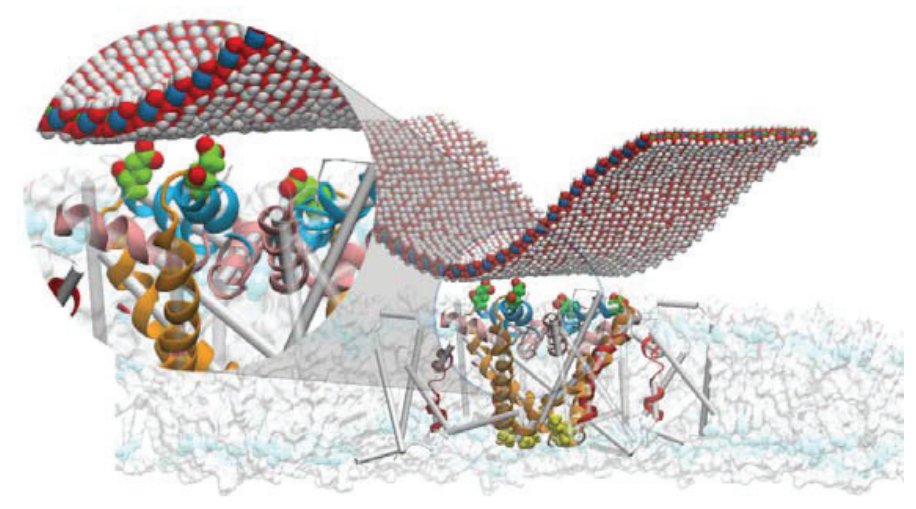

FIGURE 2. GLU residues of the external loops of the ion channel replace chlorine ions on the LDH surface during competitive adsorption, $t=0.1 \mu \mathrm{s}$ (chloride ions form an anionic shell around LDH, not shown). Colors: glutamic acid residues E189 of the turret loops - green VdW-spheres with red oxygen atoms, grey cylinders represent secondary structure of S1N—S6 segments, orange $\alpha$-helices - S6 segments, gate residues M221 (methionine)-yellow VdW-spheres, P and P2 segments of selectivity filter - pink and bright blue, respectively, voltage sensors R99-R108 in segments S4 —red helices, POPC lipidslight-grey/light-blue. Water and ions are omitted for clarity

The results of the unbiased MD simulation showed that chlorine ions formed an anionic shell around the LDH fragment, screening the electrostatic charge on it. Despite this, the nanosheet attracted the protein, and the formation of hydrogen bonds between carboxyl groups (as acceptor) of Glu189 residues of the modelled $\mathrm{Na}_{\mathrm{V}} \mathrm{Ab}$ ion channel (Fig. 2, green) and hydroxyl groups (as donor) of the Mg/Al-LDH nanosheet fragment was observed. The residues Glu189 were connected to the P and P2 segments (pink and bright blue helices, respectively), which formed a pore and a selectivity filter of the ion channel. The hydrogen bonds between the nanosheet and Glu189 could contribute to deformation of the selectivity filter's "fine structure", thereby disrupting its functioning. Since Glu189 were also connected to the S6 segments (Fig. 2, orange transmembrane $\alpha$-helices), the opposite ends of which formed the channel gate-Met221 (Fig. 2, yellow), one would expect that the bonds formed with the hydroxide surface might affect the behavior of the channel gate as well.

Moreover, during the simulation, a slow translational motion of the center of mass of the ion channel and the lipid bilayer towards the LDH fragment was observed. The estimated average velocities of these movements are $12 \mathrm{~nm} / \mu \mathrm{s}$ for the protein center of mass and $6 \mathrm{~nm} / \mu \mathrm{s}$ for the membrane center of mass. An analysis of the dynamics of $z$ coordinates of the centers of mass of voltage-sensors (VS) S4 segments (Fig. 2, red helices) showed, that voltage-sensors were moving towards LDH together with the protein, but with a lower velocity. This can be explained by the fact that VS carry a positive charge on arginine residues R99, R102, R105 и R108 [17], and the electrostatic repulsion away from the LDH promotes their slow relative displacement with respect to the whole protein toward the intracellular side of the membrane. The average relative velocity of this displacement was approximately $1.5 \mathrm{~nm} / \mu \mathrm{s}$. It may be expected that at the microsecond time scale the displacement of VS relative to the ion channel center of mass would be sufficient to start a channel gating process [19]. Furthermore, the electrostatic impact on the voltage-sensor domain (VSD) would hold the ion channels in the closed state even when the membrane is depolarized. Additionally, in the contact zone with the cell, the LDH nanosheet can come geometrically close the channel entrance, completely blocking its function. The observed binding and possible other impacts of $\mathrm{LDH}$ on the $\mathrm{Na}_{\mathrm{V}} \mathrm{Ab}$ structure and its functioning will hopefully motivate further investigations. Disruption of ion channel functionality is one of the possible ways to adversely influence tumour cell viability.

\section{SIMULATION DETAILS}

\section{The Mg/Al-LDH Nanosheet MD Models}

An LDH nanosheet having the chemical composition $\left[\mathrm{Mg}_{4} \mathrm{~A}_{12}(\mathrm{OH})_{12}\right]^{2+} \cdot 2 \mathrm{Cl}^{-} \cdot \mathrm{nH}_{2} \mathrm{O}$ was chosen for the model. The structure was built using X-ray crystallographic data from Refs. [15, 20]. Lennard-Jones (LJ), bond and angle parameters as well as partial atomic charges were taken from [16] based on the CLAYFF force field [21] with modified oxygen partial charge and LJ parameters of hydrogen atoms. In the SMD simulation series, a planar LDH 
fragment with periodic boundary conditions was used. The average surface charge density of the modeled LDH fragment was $\sigma=0.66 \mathrm{C} / \mathrm{m}^{2}$.

\section{Simulation 1. Free Energy of Flutamic Acid Anion Adsorption by $\mathrm{Mg} / \mathrm{Al}-\mathrm{LDH}$ in the Presence of $\mathrm{Cl}^{-} \mathrm{Ions}$}

The PMF profiles of the combined process of GLU adsorption-desorption on the Mg/Al-LDH surface in the presence of chloride ions were evaluated using constant velocity steered MD simulation. The velocity of the pulling procedure was $0.2 \AA / \mathrm{ns}$. Initial simulation domain dimensions were about $16 \times 18 \times 42 \AA$. Simulation was performed under the $\mathrm{NP}_{\mathrm{z}} \mathrm{T}$ conditions, where $\mathrm{P}_{\mathrm{z}}$ means that the $x y$ dimensions of the computational box were constant during SMD. The simulation box had periodic boundary conditions and contained 1133 atoms including 308 water molecules and 11 chloride anions, which at the initial time step were partially adsorbed. The net charge of the $\mathrm{Mg} / \mathrm{Al}-\mathrm{LDH}$ fragment was $12 \mathrm{e}$. To estimate the free energy of adsorption the COPFEE approach was used, which is described above. Initial (and final) position of the GLU center of mass was $z=10 \AA$, where $z=0$ is the central plane of the $\mathrm{Mg} / \mathrm{Al}-\mathrm{LDH}$ nanosheet. Total simulation time was $60 \mathrm{~ns}$.

\section{Simulation 2. The Interaction of an Mg/Al-LDH Nanosheet with an Iion Channel}

An unbiased classical MD simulation of the interaction of an $\mathrm{Mg} / \mathrm{Al}-\mathrm{LDH}$ fragment with an ion channel from the extracellular side was performed to understand if the external (turret) loops of the ion channel would adhere to the nanosheet surface, which is surrounded by shielding chloride anions. The $\mathrm{Na}_{\mathrm{V}} \mathrm{Ab}$ voltage-gated sodium channel was chosen for the model, whose atom-level structure is well-known. All-atom structural data [17, 18] were used. To provide more realistic simulation the sodium channel was embedded into a 1-palmitoyl-2-oleoyl-sn-glycero-3phosphatidyl-choline (POPC) bilayer consisting of 559 lipids in a water environment with $\mathrm{Cl}^{-}$and $\mathrm{Na}^{+}$ions. To parameterize the ion channel and the cell membrane models the CHARMM27 force field [22] was used. Initial position of the LDH fragment was $10 \AA$ above the membrane surface. The size of the computational domain was $160 \times 160 \times 140 \AA$. The total number of atoms in the system was about 340000 , total simulation time was $0.1 \mu$ s.

\section{General Simulation Parameters}

The equilibration of each system was performed in NVT conditions for about $10 \mathrm{~ns}$. The cutoff distance for nonbonded interaction was $10 \AA ̊$. The LJ potential and pairwise Coulomb interactions were smoothly shifted to zero between 8 and $10 \AA$. Periodic boundary conditions were applied along all directions. The full electrostatics was calculated, using PPPM (particle-particle particle-mesh) algorithm [23] with an accuracy of $10^{-4}$. The CHARMM27 force field was utilized for ions and water (TIP3P) parameterization. All simulations were performed at human body conditions $T=310 \mathrm{~K}$ and $p=1 \mathrm{~atm}$. The integration time step was $1 \mathrm{fs}$.

The VMD software package [24] (http://www.ks.uiuc.edu/Research/vmd) was used in model preparation and 3D-visualization of the results. The reported MD simulations were performed with the LAMMPS package (Sandia National Laboratory) [25] (http://lammps.sandia.gov/index.html).

\section{CONCLUSION}

A quick way to estimate the free energy of adsorption of small molecules based on the PMF method was proposed, which may reduce the computational cost. Employing the presented procedure the free energy of glutamic acid anion adsorption by $\mathrm{Mg} / \mathrm{Al}-\mathrm{LDH}$ nanosheet was estimated in the presence of chloride anions. The studied glutamic amino acid is a common part of proteins. For example, each of the four turret loops of voltage dependent sodium channel $\mathrm{Na}_{\mathrm{V}} \mathrm{Ab}$ contains the GLU residue. The MD simulations showed that these extracellular loops of the ion channel form hydrogen bonds with the hydroxide surface in the contact zone of LDH and the cell. Analysis of the obtained MD results allowed us to identify possible ways in which LDH may affect the voltage-gated sodium channel. These are perturbation of structure and dynamics of the selectivity filter and the gate groups as well as deactivation motions in the voltage-sensor domain and geometric covering of the channel entrance. 


\section{ACKNOWLEDGMENTS}

The present work was supported by the Russian Science Foundation (Grant No. 14-23-00096). Molecular dynamics simulations were performed using the resources of "Lomonosov-1" cluster of the Supercomputing Center (http://www.srcc.msu.su/nivc/index_engl.htm) of Lomonosov Moscow State University (MSU) [26].

The study reported in this article was conducted according to accepted ethical guidelines involving research in humans and/or animals and was approved by an appropriate institution or national research organization. The study is compliant with the ethical standards as currently outlined in the Declaration of Helsinki. All individual participants discussed in this study, or for whom any identifying information or image has been presented, have freely given their informed written consent for such information and/or image to be included in the published article.

\section{REFERENCES}

1. H. J. McClung, R. D. Beck, and P. Powers, J. Pediatr. 96(4), 769-771 (1980).

2. $\quad$ N. I. Kudrjashova, Treatment with Clay: Practical Advine and Recipes (Moscow, 1996).

3. V. N. Gorchakov, G. N. Dragun, Y. P. Kolmogorov, V. A. Smelova, L. I. Tikhonova, and Y. V. Tysjachnova, The using of SR XRF for estimation of macro-and microelement contents of biological objects at the clay treatment, Nuclear Instruments and Methods in Physics Research Section A: Accelerators, Spectrometers, Detectors and Associated Equipment 470(1), 437-440 (2001).

4. L. Mohanambe and S. Vasudevan, J. Phys. Chem. B 109, 15651-15658 (2005).

5. Z. P. Xu, et al., Int. J. Nanomed. 2, 163 (2007).

6. M. Shao, et al., J. Am. Chem. Soc. 134, 1071-1077 (2012).

7. J. H.Choy, S. J. Choi, J. M. Oh, and T. Park, Appl. Clay Sci. 36, 122-132 (2007).

8. C. Del Hoyo, Appl. Clay Sci. 36, 103-121 (2007).

9. A. N. Ay, B. Zümreoglu-Karan, A. Temel, and V. Rives, Inorg. Chem. 48, 8871-8877 (2008).

10. H. Zhang, D. Pan, K. Zou, J. He, and X. Duan, J. Mater. Chem. 19, 3069-3077 (2009).

11. D. Li, Y. T. Zhang, M. Yu, J. Guo, D. Chaudhary, and C. C. Wang, Biomater. 34, 7913-7922 (2013).

12. M. Shao, M. Wei, D. G. Evans, and X. Duan, Chem. A Eur. J. 19, 4100-4108 (2013).

13. S. Izrailev, S. Stepaniants, B. Isralewitz, D. Kosztin, H. Lu, F. Molnar, W. Wriggers, and K. Schulten, Steered molecular dynamics, in Computational Molecular Dynamics: Challenges, Methods, Ideas. Vol. 4 of Lecture Notes in Computational Science and Engineering, edited by P. Deuflhard, J. Hermans, B. Leimkuhler, A. E. Mark, S. Reich, and R. D. Skeel (Springer-Verlag, Berlin, 1998), pp. 39-65.

14. I. A. Kvasnikov, Thermodynamics and Statistical Mechanics: The Equilibrium Theory (Moscow State Univ. Publ., Moscow, 1991).

15. A. V. Arakcheeva, D. Y. Pushcharovsky, R. K. Rastsvetaeva, D. Atencio, and G. U. Lubman, Crystallography Rep. 41, 972-981 (1996).

16. A. A. Tsukanov and S. G. Psakhie, Sci. Rep. 6, 19986 (2016), doi 10.1038/srep19986.

17. J. Payandeh, T. Scheuer, N. Zheng, and W. A. Catterall, Nature 475(7356), 353-358 (2011).

18. J. Payandeh, T. M. Gamal El-Din, T. Scheuer, N. Zheng, and W. A. Catterall, Nature 486(7401), 135-139 (2012).

19. M. O. Jensen, V. Jogini, D. W. Borhani, A. E. Leffler, R. O. Dror, and D. E. Shaw, Science 336(6078), 229$233(2012)$.

20. S. V. Krivovichev, V. N. Yakovenchuk, E. S. Zhitova, A. A. Zolotarev, Y. A. Pakhomovsky, and G. Y. Ivanyuk, Mineralogical Mag. 74(5), 821-832 (2010).

21. R. T. Cygan, J.-J. Liang, and A. G. Kalinichev, J. of Phys. Chem. 108(4), 1255-1266 (2004).

22. A. D. MacKerell, D. Bashford, M. Bellott, R. L. Dunbrack, J. D. Evanseck, M. J. Field, et al., J. Phys. Chem. B 102(18), 3586-3616 (1998).

23. R. W. Hockney and J. W. Eastwood, Computer Simulation Using Particles (Adam Hilger, New York, 1989).

24. W. Humphrey, A. Dalke, and K. Schulten, J. Molec. Graphics 14, 33-38 (1996).

25. S. Plimpton, J. Computational Physics 117(1), 1-19 (1995).

26. V. A. Sadovnichy, A. Tikhonravov, Vl. Voevodin, and V. Opanasenko, "Lomonosov": Supercomputing at Moscow State University, in Contemporary High Performance Computing: From Petascale toward Exascale (Chapman \& Hall/CRC Computational Science, Boca Raton, USA, CRC Press, 2013), pp. 283-307. 